\section{Pseudoexfoliation syndrome and coronary artery ectasia}

MO Akdemir ${ }^{1}$, MR Sayin², M Armut ${ }^{1}$, I Akpinar ${ }^{2}$ and $\mathrm{SH}$ Ugurbas $^{1}$

\section{${ }^{1}$ Department of Ophthalmology, Bulent Ecevit University, School of Medicine, Zonguldak, Turkey}

${ }^{2}$ Department of Cardiology, Bulent Ecevit University, School of Medicine, Zonguldak, Turkey

Correspondence: MO Akdemir, Department of Ophthalmology, Bulent Ecevit University Medicine Faculty, Zonguldak, Turkey Tel: + 9050539746 92; Fax: + 903722610155 E-mail:doktorcun@ yahoo.com

Received: 27 July 2013 Accepted in revised form: 19 January 2014 Published online: 7 March 2014

\begin{abstract}
Aims To determine whether pseudoexfoliation syndrome is associated with coronary artery ectasia or not. Methods This cross-sectional study was undertaken at Bulent Ecevit University's Ophthalmology Department with the participation of the Cardiology Department. Eighty consecutive patients who underwent coronary angiography and were classified into two groups - those diagnosed with a normal coronary artery $(n=40)$ and those diagnosed with coronary artery ectasia ( $n=40)$-were included in the study. Pseudoexfoliation was diagnosed if typical exfoliation material was found to be present on the anterior surface of the lens, the iris, or at the pupillary border on slit-lamp examination. Age, sex, presence of pseudoexfoliation material, hypertension, diabetes mellitus, hyperlipidemia rates, cigarette smoking history, and family history of coronary artery disease were compared between the two groups. The continuous variables were compared by using the independent sample $t$-test or the MannWhitney $U$-test, and the categorical variables were compared using Pearson's $\chi^{2}$-test or Fisher's Exact $\chi^{2}$-test for the two groups. Results There was no difference in demographic data of both groups. The presence of pseudoexfoliation material was more common in patients with coronary ectasia compared with controls $[(n=21$ (52.5\%) vs $n=8$ (20\%), $P=0.005)]$. In all patients, there was a higher glaucoma rate in the patients with pseudoexfoliation syndrome $[n=7(24.1 \%), 6$ CAE $(+), 1$ CAE $(-)]$ than in the normal patients $[n=2$ (3.9\%), 2 CAE $(-)](P=0.010)$. Conclusions In this study, we found a possible association of pseudoexfoliation and coronary artery ectasia. Coronary artery ectasia patients had higher prevalence of pseudoexfoliation.
\end{abstract}

Eye (2014) 28, 594-599; doi:10.1038/eye.2014.40; published online 7 March 2014

\section{Introduction}

Pseudoexfoliation (PEX) syndrome is an agerelated disease that is rarely seen under the age of 50 years and is characterized by deposition of fibrillar material in the anterior segment of the eye and in some other tissues such as the skin and in the connective tissue portions of various visceral organs. ${ }^{1,2}$ Its prevalence varies from $0.0 \%$ (in Eskimos) to 38\% (in Navaho Indians) in different races. The reason for this variation and the exact etiology of PEX are not totally clear. ${ }^{3}$

PEX material accumulates on the lens epithelium and capsule, pupillary margin, ciliary epithelium, iris pigment epithelium, iris stroma, iris blood vessels, and subconjunctival tissue. ${ }^{4}$ At the beginning, PEX material was thought to accumulate only in ocular tissues, but then some reports revealed that it could be a systemic disorder. PEX material was found in the heart, lungs, liver, kidney, gallbladder, and walls of blood vessels, as well as in the skin and cerebral meninges. ${ }^{5,6}$

In the last decade, many studies have been performed to investigate the possible association of PEX syndrome with vascular disorders. Mitchell et al, ${ }^{7}$ Andrikopoulos et al, ${ }^{8}$ and Citirik et $a l^{9}$ found that the association between PEX syndrome and coronary artery disease was significant. Moreover, there are some studies that found a possible association of PEX syndrome with abdominal aorta aneurysm, transient ischemic attacks, and peripheral vascular disease. ${ }^{10-12}$ However, some studies did not reveal such associations. ${ }^{3,13,14}$ Thus, it is not obvious whether PEX syndrome is associated with some vascular pathology or not.

Coronary artery ectasia (CAE) is a form of atherosclerotic coronary artery disease that is defined as dilation of an arterial segment to a 
diameter at least 1.5 times that of the adjacent normal coronary artery. ${ }^{15,16}$ There are several histopathologic similarities between CAE and atherosclerosis. The pathogenesis of CAE is thought to be a different form of atherosclerotic disease and vascular remodeling. However, a definite link between atherosclerosis and CAE has not been confirmed. ${ }^{17}$

The aim of this study was to determine whether PEX is associated with CAE or not.

\section{Materials and methods}

A cross-sectional study was undertaken at Bulent Ecevit University's ophthalmology department with the participation of the cardiology department. Eighty consecutive patients who underwent coronary angiography at the cardiology department and were either diagnosed as normal $(n=40)$ or age- and sex-matched patients who were diagnosed with CAE ( $n=40)$ were included in the study. Informed consent was obtained from each participant and the study was conducted in accordance with the Declaration of Helsinki Principles. The study was approved by the Clinical Research Ethics Committee of Bulent Ecevit University. Upon power analysis, the estimated sample size was calculated as 35 patients for each group, which results in a maximum 5\% chance of type I error and $80 \%$ power.

Coronary angiography was performed with Judkins' technique and without the use of nitroglycerin. Coronary artery diameters were measured by computerized quantitative angiography. The largest diameters were considered in the segments. CAE was defined as the expansion of the coronary artery by 1.5 times or more as compared with the normal coronary artery segment. Normal segment was defined as a segment without CAE and coronary artery stenosis. CAE associated with coronary stenosis of $20 \%$ or more was excluded from the study. Normal coronary arteries were defined as those with the absence of any irregularities on their surface. Coronary arteries that had ectasia were also evaluated in the CAE $(+)$ group. Comparison of vessel involvement by type and number between the PEX $(+)$ and the PEX (-) patients in the CAE $(+)$ group was carried out.

All the patients were evaluated by the same ophthalmologist (MOA) who was blinded to the results of angiography. The patients who had a history of ophthalmic surgery, ocular trauma, uveitis, any ophthalmic disease preventing the view of the anterior chamber detail, and were diagnosed with any disease other than CAE by angiography were excluded from the study. A detailed medical history was recorded and complete ophthalmological examinations were performed. Complete ophthalmological examination included visual acuity with Snellen chart, intraocular pressure measurement with applanation tonometry, biomicroscopy, gonioscopy, and fundus and visual field examinations. PEX was diagnosed if typical exfoliation flakes were found to be present on the anterior surface of the lens or the iris, or at the pupillary border by slit-lamp examination after dilating the pupil with $1 \%$ tropicamide. ${ }^{18}$ Glaucoma was diagnosed by the Humphrey 30-2 full-threshold visual field test, IOP measurement, optic nerve head examination with a 90 diopter (D) lens, and gonioscopic examination. All glaucoma patients had open-angle, glaucomatous optic disc, and visual field changes with an IOP greater than $21 \mathrm{~mm} \mathrm{Hg}$.

All patients' diabetes mellitus (DM), arterial hypertension (HT), hyperlipidemia (HL), and smoking history, with family history of coronary artery disease (CAD) were recorded. The patients were considered to have HT if they had previously known HT, were on antihypertensive therapy, or had a systolic blood pressure of $\geq 140 \mathrm{~mm} \mathrm{Hg}$ and a diastolic blood pressure of $\geq 90 \mathrm{~mm} \mathrm{Hg}$, which were calculated as the mean of two measurements taken on each arm. Patients were considered to have type II DM if they were previously diagnosed and treated for DM, and/or had a fasting blood glucose level of $\geq 126 \mathrm{mg} / \mathrm{dl}$. The patients were considered to have HL if they had a fasting total cholesterol level $\geq 200 \mathrm{mg} / \mathrm{dl}$, a fasting low-density lipoprotein level $\geq 160 \mathrm{mg} / \mathrm{dl}$, a fasting triglyceride level $\geq 200 \mathrm{mg} / \mathrm{dl}$, or were on anti-hyperlipidemia therapy. The family history of CAD was considered as a history of CAD or sudden cardiac death in a first-degree relative before the age of 55 years for men and 65 years for women.

Statistical analyses were performed with SPSS 18.0 software (SPSS Inc., Chicago, IL, USA). The distribution of data was determined by the Shapiro-Wilks test. The continuous variables were expressed as mean \pm SEM or median (minimum-maximum), and the categorical variables as frequency and percent. The continuous variables were compared by using the Independent Sample $t$-test or the Mann-Whitney $U$-test and the categorical variables were compared using Pearson's $\chi^{2}$-test or Fisher Exact $\chi^{2}$-test for the two groups. $P$-value less than 0.05 was considered statistically significant for all tests.

We certify that all applicable institutional and governmental regulations concerning the ethical use of human volunteers were followed during this research.

\section{Results}

There was no statistically significant difference between the ages and genders of the CAE $(+)$ and the CAE (-) 
groups $(P=0.137, P=0.180)$. The patient characteristics are summarized in Table 1. The presence of PEX material was higher in the CAE $(+)$ group. Twenty-one $(52.5 \%)$ out of $40 \mathrm{CAE}(+)$ patients and $8(20 \%)$ out of the $40 \mathrm{CAE}$ $(-)$ patients were diagnosed as $\operatorname{PEX}(+)$. This difference was statistically significant $(P=0.005)$.

We also evaluate the laterality of eye involvement by PEX material. In the CAE ( - ) group, PEX material was diagnosed in the right eyes of two patients, in the left eyes of two patients, and in bilateral eyes of four patients. In the CAE $(+)$ group, PEX material was diagnosed in the right eyes of nine patients, in the left eyes of four patients, and in bilateral eyes of eight patients. There was no statistically significant difference between the two groups with regard to eye involvement in PEX $(P=0.665)$.

There was higher glaucoma rate in the PEX $(+)$ group. In the PEX $(+)$ group, $7(24.1 \%)$ [6 CAE $(+)$, $1 \mathrm{CAE}(-)]$ of 29 patients, whereas in the PEX $(-)$ group $2(3.9 \%)$ [2 CAE $(-)$ ] of 51 patients were diagnosed with glaucoma. This difference was statistically significant $(P=0.010)$.

Coronary artery ectasia was diagnosed in the left anterior descending artery (LAD), circumflex artery (Cx), and/or the right coronary artery (RCA). There was no particular association between PEX and the type and number of vessel involvement in the CAE $(+)$ group. In the CAE $(+)$ group, 12 patients had 1-vessel, 15 patients had 2-vessel, and 13 patients had 3-vessel involvement. In the CAE and PEX $(+)$ groups, 7 patients had 1-, 9 patients had 2-, and 5 patients had 3-vessel involvement. In the CAE and PEX (-) groups, 6 patients had 1-, 6 patients had 2-, and 7 patients had 3 -vessel involvement. In the CAE $(+)$ group, the difference between the number of vessel involvements in the PEX $(+)$ and the PEX (-) groups was not statistically significant $(P=0.347)$. In the CAE $(+)$ group, 29 (16 $\operatorname{PEX}(-)$, $13 \operatorname{PEX}(+), P=0.221)$ patients had LAD ectasia, 28 patients (15 PEX $(-)$, $13 \operatorname{PEX}(+)$, $P=0.407)$ had Cx ectasia, and 24 patients (10 PEX $(-)$, $14 \operatorname{PEX}(+), P=0.561)$ had RCA ectasia. There was no statistically significant difference between the types of vessel involvement in the PEX (+) and the PEX (-) patients of the CAE $(+)$ group.

HT was diagnosed in 24 patients of the CAE $(-)$ group and in 26 patients of the CAE $(+)$ group $(P=0.817)$. DM was diagnosed in 6 patients of the CAE $(-)$ group and in 11 patients of the CAE $(+)$ group $(P=0.247)$. HL was diagnosed in 9 patients of the CAE $(-)$ group and in 12 patients of the CAE $(+)$ group $(P=0.611)$. Cigarette smoking history was found in 11 patients of the CAE ( -$)$ group and in 16 patients of the CAE $(+)$ group $(P=0.344)$. CAD history was found in 17 patients of the CAE $(-)$ group and in 17 patients of the CAE $(+)$ group $(P=1.000)$. The differences between the distribution of subjects in the CAE $(+)$ and the CAE $(-)$ groups by systemic illnesses (HT, DM, and HL), cigarette smoking, and CAD family history were not statistically significant.

The differences between the distribution of subjects in the PEX $(+)$ and the PEX $(-)$ groups by systemic illnesses (HT, DM, and HL), cigarette smoking, and CAD history were also not statistically significant (Table 2).

\section{Discussion}

PEX syndrome is characterized by the production and progressive accumulation of fibrillar extracellular material in many ocular tissues. Etiology and pathophysiology of the PEX syndrome are still not obvious; however, PEX is thought to be a systemic biochemical process with environmental and genetic risk factors. ${ }^{19}$ LOXL1 gene that is responsible for crosslinking of elastin was found to be related with the PEX syndrome. ${ }^{20}$ Moreover, molecular, biological, and biochemical evidence showed that it is a type of stressinduced elastic microfibrillopathy. ${ }^{21}$

It is a very important entity for ophthalmology in which the syndrome causes severe, chronic open-angle glaucoma, angle-closure glaucoma, blood aqueous barrier impairment, lens subluxation, and increased complications during cataract surgery. ${ }^{4,22}$ The prevalence

Table 1 Patient characteristics

\begin{tabular}{lccc}
\hline & CAE $(-) \mathrm{n}=40$ & $C A E(+) \mathrm{n}=40$ & P-value \\
\hline Age, mean \pm SEM & $62.2 \pm 3.9$ & $64.2 \pm 2.0$ & 0.137 \\
Gender $(\mathrm{n}(\%))$ & & & \\
$\quad$ Female & $16(40)$ & $23(57.5)$ & 0.180 \\
$\quad$ Male & $24(60)$ & $17(42.5)$ & \\
\hline
\end{tabular}

Abbreviation: CAE, coronary artery ectasia.

Table 2 Comparison of HT, DM, HL, CS, CAD family history rates between PEX $(-)$ and $\operatorname{PEX}(+)$ patients

\begin{tabular}{lccr}
\hline & $\begin{array}{c}\text { PEX }(-) \\
(\mathrm{n}=51) \mathrm{n}(\%)\end{array}$ & $\begin{array}{c}\text { PEX }(+) \\
(\mathrm{n}=29) \mathrm{n}(\%)\end{array}$ & P-value \\
\hline HT & $34(66.7)$ & $16(55.2)$ & 0.435 \\
DM & $13(25.5)$ & $4(13.8)$ & 0.345 \\
HL & $16(31.4)$ & $5(17.2)$ & 0.264 \\
CS & $17(33.3)$ & $10(34.5)$ & 1.000 \\
CAD family history & $17(45.1)$ & $17(37.9)$ & 0.698 \\
\hline
\end{tabular}

Abbreviations: CAD, coronary artery disease; CS, cigarette smoking; $\mathrm{DM}$, diabetes mellitus; HL, hyperlipidemia; HT, hypertension. 
of PEX glaucoma varies from country to country depending on race and age. The prevalence is as high as $\sim 20 \%$ in Finland and over 25\% in Iceland, but is only $\sim 5 \%$ in parts of Denmark. ${ }^{23}$ Yalaz et al ${ }^{24}$ examined 1356 people who were over 40 years of age and found the incidence of PEX syndrome to be $11.2 \%$ in Turkey. Moreover, they found that the percentage of glaucoma in patients with PEX syndrome was $34.3 \%$. In this study, we found glaucoma percentage in the PEX $(+)$ patients to be $24.1 \%$ and $3.9 \%$ in the PEX ( - ) patients. This difference was statistically significant as expected $(P=0.01)$.

PEX material can be produced by various intraocular cell types such as the preequatorial lens epithelium, nonpigmented ciliary epithelium, trabecular endothelium, corneal endothelium, vascular endothelial cells, and all cell types of the iris. ${ }^{25}$ In the last two decades, it has been shown that PEX material could be identified in several tissues. It was found that PEX material was primarily found in connective tissue portions of visceral organs, often in the periphery of blood vessels. It was thought to originate from connective tissue fibroblasts, smooth and striated muscle cells, and heart muscle cells. With these findings, in addition to long-known processes in ocular tissues, it has been shown to be a systemic disorder that causes increased cardiovascular and cerebrovascular morbidity. ${ }^{26,27}$ However, there is no consensus about the relationship between some vascular disorders and the PEX syndrome.

Speckauskas et al ${ }^{19}$ conducted a recent study, including 1065 participants aged 45-72 years, aiming to determine the prevalence of PEX syndrome and its association with ischemic heart disease (IHD), HT, and DM. The rates of IHD, HT, and DM were higher in the PEX group but only the HT rates reached statistical significance $(P=0.017)$. Myocardial infarction was observed only in $2 \%$ and $1.6 \%$ of the PEX and non-PEX patients, respectively $(P=0.67)$. DM was found in $6.8 \%$ patients of the non-PEX group and in $7.4 \%$ patients of the PEX group $(P=0.82)$. However, HT was found more in the PEX group (76.8\%) than in the non-PEX $(64.9 \%)$ group. This difference was statistically significant $(P=0.017)$.

In another study, 490 patients who underwent coronary angiography were evaluated. Patients were examined by an ophthalmologist and the existence of PEX material was evaluated. Emiroglu et al diagnosed coronary artery disease in 387 patients, and PEX was found in 20 (5.2\%) CAD patients and in $4(3.9 \%)$ normal angiography patients $(P>0.05)$. They concluded that there is no significant relationship between PEX and CAD. ${ }^{13}$

However, some other studies demonstrated this relationship. Citirik et al compared the patients with
CAD proven by coronary angiography with 50 controls with normal coronary angiographic findings in terms of PEX, other vascular diseases, and retinal vascular findings. In total, 28 out of $50 \mathrm{CAD}(+)$ patients and 12 out of 50 CAD (-) patients had PEX. This finding was statistically significant $(P=0.001)$. Moreover, they found DM and HT were also more prevalent in patients with PEX but the results were not statistically significant. ${ }^{9}$ There is also no consensus on whether these diseases were associated with PEX or not. ${ }^{28}$ In a recent study performed in Greek patients, Andrikopoulos also found that pseudoexfoliation is a risk factor for CAD and glaucoma. ${ }^{8}$ We did not find any correlation between PEX syndrome and HT, DM, HL, cigarette smoking history, or coronary artery disease family history.

Sekeroglu et $a l^{29}$ also showed the relationship between ischemic heart disease and PEX. They investigated 1480 patients who were scheduled for cataract surgery. The patients underwent a comprehensive systemic and eye examination. They found that $242(16.4 \%)$ patients had PEX syndrome and that the only systemic disease associated with PEX was ischemic heart disease (OR:1.49).

Another vascular pathology that is considered to have a possible association with PEX is abdominal aorta aneurysm. Similar to ischemic heart disease, there is no obvious link between abdominal aorta aneurysm and PEX. Schumacher et al proposed that their study suggests an association between aneurysm of the abdominal aorta and PEX syndrome; however, Hietanen et al concluded that the prevalence of exfoliation syndrome was similar in patients with abdominal aorta aneurysm and in the general population. ${ }^{10,14}$ In a large national cross-sectional comparison study consisting of 6000 patients with PEX, French et $a l^{30}$ found correlations between PEX and several cardiovascular diseases such as abdominal aorta aneurysm, chronic ischemic heart disease, and cardiomyopathy.

Different results established about the PEX syndrome even in the same country make the disease hard to understand. There are still a lot of questions that have to be answered about pseudoexfoliation syndrome. In this study, we found a possible relationship between PEX and CAE. The CAE patients had higher prevalence of PEX, which is statistically significant. However, no association was found between $\mathrm{HT}, \mathrm{HL}$, or DM with PEX syndrome in this study. As far as we know, this is the first study that shows the relationship between CAE and PEX syndrome. Future studies with larger populations are needed to clarify the relationship and the systemic characteristics of PEX syndrome. 
Summary

What was known before

- Pseudoexfoliation syndrome is related with ischemic heart disease, HT, and abdominal aorta aneurysm.

What this study adds

- An unknown relationship between pseudoexfoliation syndrome and coronary artery ectasia was found with this study.

- Pseudoexfoliation syndrome is also related with coronary artery ectasia.

\section{Conflict of interest}

The authors declare no conflict of interest.

\section{Acknowledgements}

This research received no specific grant from any funding agency in the public, commercial, or not-for-profit sectors. The study was approved by the Clinical Research Ethics Committee of Bulent Ecevit University.

\section{Author contributions}

MOA: conception and design, acquisition of data, analysis and interpretation, drafting the article. MRS: conception and design, acquisition of data, analysis and interpretation, drafting article. MA: literature research, acquisition of data. IA: acquisition of data. SHU: revising the manuscript critically for important intellectual content and final approval of the version to be published.

\section{References}

1 Streeten BW, Dark AJ, Wallace RN. Pseudoexfoliative fibrillopathy in the skin of patients with ocular pseudoexfoliation. Am J Ophthalmol 1990; 110: 490-499.

2 Streeten BW, Li ZY, Wallace RN. Pseudoexfoliative fibrillopathy in visceral organs of a patient with pseudoexfoliation syndrome. Arch Ophthalmol 1992; 110: 1757-1762.

3 Shrum KR, Hattenhauer MG, Hodge D. Cardiovascular and cerebrovascular mortality associated with ocular pseudoexfoliation. Am J Ophthalmol 2000; 129: 83-86.

4 Ritch R. Exfoliation syndrome. Curr Opin Ophthalmol 2001; 12: 124-130.

5 Vesti E, Kivela T. Exfoliation syndrome and exfoliation glaucoma. Prog Retin Eye Res 2000; 19: 345-368.

6 Schlötzer-Schrehardt U, Naumann GO. Ocular and systemic pseudoexfoliation syndrome. Am J Ophthalmol 2006; 141: 921-937.

7 Mitchell P, Wang JJ, Smith W. Association of pseudoexfoliative syndrome with increased vascular risk. Am J Ophtalmol 1997; 124: 684-687.

8 Andrikopoulos GK, Mela EK, Georkakopoulos CD, Papadopoulos GE, Damelou AN, Alexopoulos DK et al.
Pseudoexfoliation syndrome prevalence in Greek patients with cataract and its association to glaucoma and coronary artery disease. Eye 2009; 23: 442-447.

9 Citirik M, Acaroglu G, Batman C, Yildiran L, Zilelioglu O. A possible link between the pseudoexfoliation syndrome and coronary artery disease. Eye 2007; 21: 11-15.

10 Schumacher S, Scholötzer-Schrehardtz U, Martus P, Lang W, Naumann GO et al. Pseudoexfoliation syndrome and aneurysms of the abdominal aorta. Lancet 2001; 357: 359-360.

11 Repo LP, Terasvirta ME, Koivisto KJ. Generalized transluminance of the iris and frequency of pseudoexfoliation syndrome in the eyes of transient ischemic attack patients. Ophthalmology 1993; 100: 353-355.

12 Praveen MR, Shah SK, Vasavada AR, Diwan RP, Shah SM, Zumkhawala BR et al. Pseudoexfoliation as a risk factor for peripheral vascular disease: a case control study. Eye 2011; 25: 174-179.

13 Emiroglu MY, Coskun E, Karapinar H, Capkın M, Kaya Z, Kaya $\mathrm{H}$ et al. Is pseudoexfoliation syndrome associated with coronary artery disease? N Am J Med Sci 2010; 2: 487-490

14 Hietanen J, Soisalon-Soininen S, Kivela T, Tarkkanen A. Evaluation of the clinical association between exfoliation syndrome and abdominal aortic aneurysm. Acta Ophthalmol Scand 2002; 80: 617-619.

15 Mavrogeni S. Coronary artery ectasia:from diagnosis to treatment. Hellenic J Cardiol 2010; 51: 158-163.

16 Hartnell GG, Parnell BM, Pridie RB. Coronary artery CAE. Its prevalence and clinical significance in 4993 patients. Br Heart J 1985; 54: 392-395.

17 Antoniadis AP, Chatzizisis YS, Giannoglou GD. Pathogenetic mechanisms of coronary ectasia. Int J Cardiol 2008; 130: 335-343.

18 Hietanen J, Soisalon-Soininen S, Kivela T, Tarkannen A. Evaluation of the clinical association between exfoliation syndrome and abdominal aortic aneurysm. Acta Ophthalmol Scand 2002; 80: 617-619.

19 Speckauskas M, Tamosiunas A, Jasinskas V. Association of ocular pseudoexfoliation syndrome with ischaemic heart disease, arterial hypertension and diabetes mellitus. Acta Ophthalmol 2012; 90: 470-475.

20 Schlötzer-Schrehardt U. Molecular pathology of pseudoexfoliation syndrome/glaucoma-New insights from LOXL1 gene associations. Exp Eye Res 2009; 88: 776-785.

21 Ovodenko B, Rostagno A, Neubert TA, Shetty V, Thomas S, Yang A et al. Proteomic analysis of exfoliation deposits. Invest Ophthalmol Vis Sci 2007; 48: 1447-1457.

22 Ritch R. Exfoliation syndrome: the most common identifiable cause of open-angle glaucoma. J Glaucoma 1996; 3: 176-178.

23 Desai MA, Lee RK. The medical and surgical management of pseudoexfoliation glaucoma. Int Ophthalmol Clin 2008; 48: 95-113.

24 Yalaz M, Othman I, Nas K, Eroglu A, Homurlu D, Cikintas $\mathrm{Z}$ et al. The frequency of pseudoexfoliation syndrome in the eastern mediterranean area of Turkey. Acta Ophthalmol 1992; 70: 209-213.

25 Naumann GOH, Schlötzer-Schrehardt U, Küchle M. Pseudoexfoliation syndrome for the comprehensive ophthalmologist:intraocular and systemic manifestations. Ophthalmology 1998; 105: 951-968.

26 Schlötzer-Schrehardt U, Naumann GOH. Ocular and systemic pseudoexfoliation syndrome. Am J Ophthalmol 2006; 141: 921-937. 
27 Scholotzer-Schrehardtz UM, Koca MR, Naumann GOH, Volkhol H. Pseudoexfoliation syndrome; ocular manifestation of a systemic disorder? Arch Ophthalmol 1992; 110: 1752-1756.

28 Tarkkanen A. Is exfoliation syndrome a sign of systemic vascular disease? Acta Ophthalmol 2008; 86: 832-836.
29 Sekeroglu MA, Bozkurt B, Irkec M, Ustunel S, Orhan M, Saracbasi O. Systemic associations and prevalence of exfoliation syndrome in patients scheduled for cataract surgery. Eur J Ophthalmol 2008; 18: 551-555.

30 French DD, Margo CE, Harman LE. Ocular pseudoexfoliation and cardiovascular disease: A national cross section comparison study. N Am J Med Sci 2012; 4: 468-473. 